\title{
The innate function of circular RNAs
}

\section{Following viral infection, PKR is activated through the degradation of circRNAs by RNase $\mathrm{L}$}

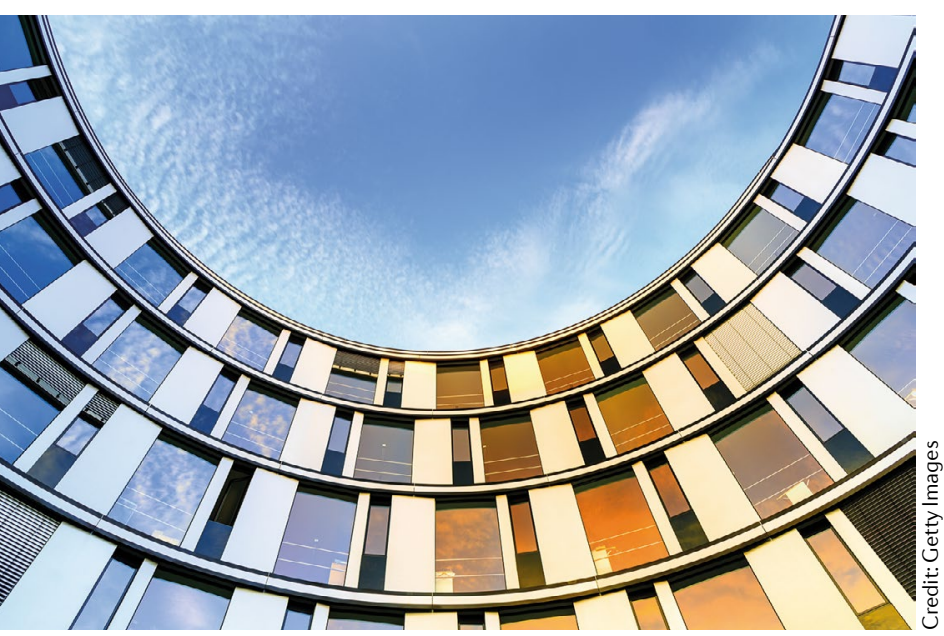

Circular RNAs (circRNAs) are produced by the infrequent backsplicing of exons. Some circRNAs have been shown to bind microRNAs and proteins and modulate gene expression, but the functional repertoire of circRNAs remains mostly uncharacterized. Now, Liu et al. show that circRNAs can prevent aberrant activation of the innate immune response to viral infection.

CircRNAs are implicated in the regulation of innate immunity, but the molecular basis of their involvement remains unclear, partly because little is known about circRNA turnover in cells. Circularity endows circRNAs with resistance to RNA decay machineries, which mostly use exonucleases. The authors therefore set out to characterize circRNA turnover in different stress conditions. Of these, treatment of human cell lines with poly(I:C), which mimics pathogenic double-stranded RNA (dsRNA) or viral infection, strongly and rapidly reduced circRNA levels (in HeLa cells from $\sim 10,000$ circRNA copies to $\sim 1,500$ copies) through a mechanism involving RNA degradation. Importantly, the reduction in circRNA levels was also observed in cells infected with the RNA virus encephalomyocarditis virus (EMCV).

Cell infection by RNA viruses (including ECMV) or treatment with poly(I:C) activate the cytoplasmic endoribonuclease RNase L, which then catalyses the degradation of viral and cellular RNA. The catalytic activity of RNase $\mathrm{L}$ was required for the reduction in circRNA levels upon poly(I:C) treatment, and synthetic activation of RNase L caused global circRNA degradation. Thus, RNase L is the main enzyme degrading circRNA upon viral infection.

To understand how circRNA degradation might modulate innate immunity responses, the authors searched for factors that associate with circRNAs. The interferonresponse-inducing antiviral factor, double-stranded RNA-activated protein kinase (PKR), was found to preferentially bind to circRNA over cognate, sequence-identical linear RNA. Strikingly, the addition of purified circRNAs dampened the activation of PKR.

PKR is activated through selfphosphorylation upon binding to dsRNA longer than $33 \mathrm{bp}$, but is repressed by the binding of shorter dsRNA. To understand the basis of the PKR repression by circRNAs, the structures of paired circular and linear RNAs were compared. The analysis revealed that most, albeit not all, circRNAs tend to form imperfect RNA duplexes of 16-26 bp. Thus, circRNAs tend to form dsRNA with the potential to repress PKR.

In cells mildly overexpressing duplex-forming circRNAs $(\sim 5,000$ extra copies per cell), but not in cells overexpressing non-duplexforming circRNAs or cognate linear sequences, PKR activity following poly(I:C) treatment was markedly reduced. Subsequently,
RNA immunoprecipitation showed that PKR preferentially associates with dsRNA-forming circRNAs. Overexpression of a dsRNA-forming circRNA strongly blocked PKR activation also during EMCV infection and led to increased viral replication. Thus, PKR-circRNA interactions prevent PKR activation in uninfected cells; activation of the PKR innate immune response following viral infection requires extensive and rapid circRNA degradation by RNase L.

Given their function in innate immunity, what could be the physiological relevance of circRNAs? Aberrant activation of innate immunity responses can contribute to the development autoimmune diseases. In blood cells of individuals with systemic lupus erythematosus (SLE), RNase L and PKR were activated, and the number and expression of circRNAs were reduced compared with those of healthy individuals. Importantly, overexpression of a dsRNA-forming circRNA in SLE blood cells reduced PKR activity and the expression of SLE-relevant genes.

In summary, in uninfected cells, dsRNA-forming circRNAs interact with PKR and prevent aberrant activation of innate immunity. Following viral infection, PKR is activated through the degradation of circRNAs by RNase L. Owing to their stability, the use of circRNAs as therapeutic agents for innate immunity-associated diseases should be pursued. Furthermore, it would be useful to study the interactions of circRNAs with various RNA-binding proteins.

Eytan Zlotorynski

ORIGINAL ARTICLE Liu, C.-X. et al. Structure and degradation of circular RNAs regulate PKR activation in innate immunity. Cell 177, 865-880 (2019) 